\title{
Application of Parallel Redundancy in a Wi-Fi- based WNCS using OPNET
}

Mostafa Hendawy, Mohamed ElMansoury, Karim N. Tawfik, Mohamed M. ElShenawy, Alia H. Nagui, Ahmed T. ElSayed

Electronics Engineering Department

American University in Cairo Cairo, Egypt

\{mhindawi; mansoury; karimnabil; shinnawy7; alianagui; a_t\}@aucegypt.edu

Hassan H. Halawa, Ramez M. Daoud, Hassanein H. Amer Electronics Engineering Department

American University in Cairo Cairo, Egypt

\{hhhalawa; rdaoud\}@ieee.org, hamer@aucegypt.edu

\begin{abstract}
A powerful approach to improve the performance of wireless communication is the parallel redundant transmission with dual-radio wireless devices. To further verify this approach in this work, an OPNET simulation is performed on a startopology WNCS workcell with 30 sensor and actuator pairs that are equipped with dual-radios for parallel redundancy. The applied wireless simulation model is based on IEEE 802.11g (WiFi) standard and a quantitative analysis of the effect of interference in an industrial environment, is presented. This study proved that parallel redundancy improves system performance under different interference environments.
\end{abstract}

Keywords - diversity, parallel redundancy, wi-fi, 802.11, opnet

\section{INTRODUCTION}

Control networks are mainly intended for the communication of very small packets at a high transmission and reception rate $[1,2]$. Since these networks are usually designed for a large number of nodes that involve real-time applications, timely packet reception is very important and hence, a high level of reliability and minimal losses must always be ensured [3]. Different network applications may use different protocols and depending on the application, it is determined whether retransmission is necessary [4]. In order to ensure high performance and reliability of control networks, deterministic network communication protocols like CAN and Profibus are utilized $[1,5]$. IEEE 802.3 Ethernet was also introduced as a communication protocol for wired Networked Control Systems (NCSs) [3, 5, 6].

Wireless Networked Control Systems (WNCSs) became an important research topic for industrial applications due to several advantages that include less cabling and ease of installation and maintenance. One of several WNCS solutions available today is Wireless Interface for Sensors and Actuators (WISA) that used a modified version of IEEE 802.15.1 (Bluetooth) as a communication protocol between sensors, actuators, and the controller. WISA can also provide wireless power for sensors and actuators.

\author{
Markus Rentschler \\ Business Unit Networking \\ Balluff GmbH \\ Germany \\ Markus.Rentschler@balluff.de
}

Hany M. ElSayed
Electronics and Communications Department
Cairo University
Giza, Egypt
helsayed@ieee.org

Other alternative WNCS solutions were discussed. Reference [7] proposed a system that applied IEEE 802.11b $\mathrm{Wi}-\mathrm{Fi}$ [8] and Ethernet protocols without modifications in order to model an industrial WNCS. The system in [7] used those presented in $[9,10]$ as its benchmark where $\mathrm{Wi}-\mathrm{Fi}$, in particular, was chosen over Bluetooth and ZigBee [11, 12] because of its superiority in terms of wider range and larger data rate. The proposed system produced an improvement in meeting timing requirements when compared to $[9,10]$, even with utilizing standard off-the-shelf equipment in the presence of external interference. The performance and tolerance to interference of the workcell model proposed in [7] was further enhanced in [13]. This was achieved via adjusting the delay constraint conditions while maintaining the overall end-to-end delay requirement of [9].

The performance of a wireless communication system can be significantly improved by applying diversity, which is basically the redundant transmission of information over stochastically uncorrelated channels [14]. A possible diversity scheme utilizes parallel redundancy in the space and frequency domains, which is able to yield specific gains especially in packet transmission schemes [15]. A recently presented example is Parallel Redundant WLAN (PRPWLAN), which experimentally used the Parallel Redundancy Protocol (PRP) according to IEC 62439-3 [16] as splitter and combiner units on the Ethernet level and could yield significant improvements [17, 18]. This was further verified through the OPNET simulations studied in [19]. The mentioned PRP principle can also directly be applied on the wireless system's MAC packet transmission layer, achieving simpler implementations in dual-radio devices, an approach that will be followed in this work.

The assessment of interference on real-time wireless communication systems has received widespread research interest. Reference [20] studied the effect of interference on real-time communication in IEEE 802.11-based mesh networks. 
In this paper, the approach is further verified with an OPNET simulation model of a star-topology WNCS work cell with 30 sensor-actuator pairs that are equipped with dualradios for parallel redundancy. The concept of PRP that was used in [19] was applied to the full industrial workcell described in [13] in order to investigate the performance improvements achievable through the use of PRP. It will be shown that, even under interference, the PRP system performs significantly better than a corresponding single channel system.

This paper is structured as follows: In Section II, related previous work is mentioned. Section III describes the OPNET model specification, Section IV the analysis of the simulation results, whereas Section V concludes this work.

\section{PREVIOUS WORKS}

Parallel Redundant Transmission is a concept that has been introduced several times in the literature. Reference [19] showed that parallel redundant transmission results in a substantial improvement over transmission on one channel. The model in [19] contained one sensor-actuator pair that was communicating on two non-interfering IEEE $802.11 \mathrm{~g}$ channels. Several interference sources were utilized and PRP proved superior in all cases. In [19], every packet is duplicated and sent by the sensor over the two non-interfering channels. The packet that is received first by the controller is utilized and the later arriving duplicate is eliminated. Interference was applied in three different cases, on one channel only, on the other symmetric channel, and on both channels simultaneously. For the purpose of this study, a similar approach will be taken for these three cases on a different physical model.

The model applied in [13] simulates a typical factory environment that has an array of 30 sensors and 30 actuators that communicate, through a central controller, using IEEE $802.11 \mathrm{~b}$. Each 15 sensor-actuator pairs use one $802.11 \mathrm{~b}$ channel with a data rate of $11 \mathrm{Mbps}$. In [13], a single packet is sent from its respective sensor to the workcell's controller. The controller then forwards the packet to the designated actuator. The User Datagram Protocol (UDP), rather than Transmission Control Protocol (TCP), was used for the transmission of control information due to less network congestion from acknowledgements [21]. Reference [22] improved upon the model described in [7] by changing the employed communication protocol to IEEE 802.11g. The increased throughput allowed for the implementation of controller fault-tolerance across multiple concatenated workcells.

\section{OPNET MODEL SPECIFICATIONS}

In this section, PRP-WLAN will be introduced in the context of the workcell described in [13]. The proposed model will be developed and simulated on OPNET Network Modeler.

\section{A. General Model Description}

The simulated model uses $802.11 \mathrm{~g}$ with a data rate of $54 \mathrm{Mbps}$ similar to the workcell described in [22], instead of the previously-used $802.11 \mathrm{~b}$ model. Similar to [13], 30 sensor-actuator pairs communicate simultaneously in the workcell. The main difference is that one $802.11 \mathrm{~g}$ channel is allocated to the whole workcell, instead of two $802.11 \mathrm{~b}$ channels. This is possible due to the higher data rate offered by $802.11 \mathrm{~g}$. Moreover, PRP is implemented by sending every packet on another non-interfering $802.11 \mathrm{~g}$ channel. This would assure that the channels used, namely 1 and 6 , would work separately without overlap. A sensor sends the same packet over two different channels to the controller. This controller would then forward the received packet to the respective actuator. The actuator uses the packet that arrives first and discards the duplicate packet. Fig. 1 shows the workcell that will be used for this study.

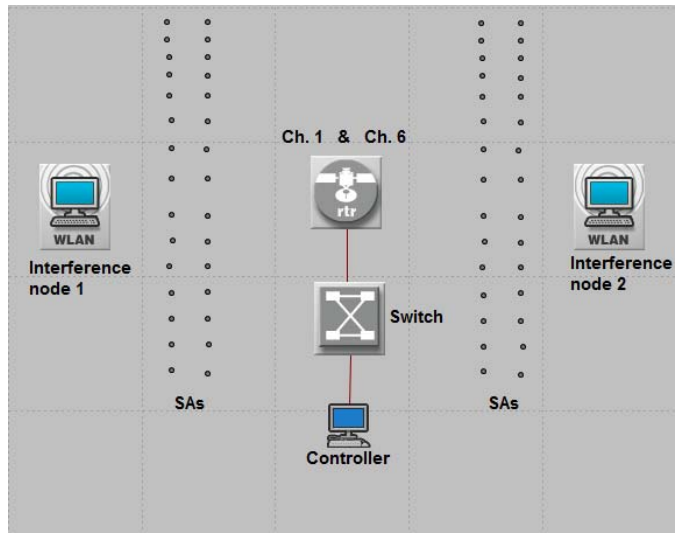

Fig. 1. Model Overview

Table I shows the general model specifications that were used for the simulations. These parameters were kept constant for the entirety of this study.

TABLE I. MODEL SPECIFICATIONS

\begin{tabular}{|l|l|}
\hline \multicolumn{1}{|c|}{ Parameter } & \multicolumn{1}{|c|}{ Value } \\
\hline Sampling Period & $40 \mathrm{~ms}$ \\
\hline Transmission Power & $0.03 \mathrm{Watts}$ \\
\hline Transmission Data Rate & $54 \mathrm{Mbps}$ \\
\hline Cell Dimensions & $3 \mathrm{~m} \times 3 \mathrm{~m}$ \\
\hline Sensor to Actuator (SA) Distance & $0.3 \mathrm{~m}$ \\
\hline SA Pair Separation Distance & $0.2 \mathrm{~m}$ \\
\hline System Transport Layer Protocol & TCP \\
\hline
\end{tabular}

The sampling period in Table I represents a deadline that every packet has to meet in order to be considered succesfully sent. This value was taken from [13] as the deadline of the system, therefore it cannot be changed. Additionally, it is a reasonable value in this kind of industrial communication.

In order to establish a benchmark for communication, the model was simulated in an interference-free environment first and then compared to later simulations with added interference. For each scenario, 33 seeds are simulated, and all simulation results are subjected to a $95 \%$ confidence analysis.

\section{B. Interference-Free PRP Model}

The two channels are expected to exhibit similar behavior since the transmission data rates and control payloads are identical over the symmetrical non-interfering channels. This 
model is then used to study the behavior of the system utilizing PRP.

\section{PRP Model under Interference}

The main purpose of the OPNET simulations is to study the performance benefits offered by PRP in an industrial system especially under the effect of external interference. Channel congestion for one channel is achieved by adding one laptop pair (interference nodes in Fig. 1) that is positioned in order to maximize interference on the workcell as in [7, 13]. An additional laptop pair was introduced to add interference on both channels simultaneously. Each laptop pair communicated on either one of the two channels used by the sensor-actuator pairs. Both laptop pairs utilized the File Transfer Protocol (FTP). In the simulated interference models, interference was quantified by the FTP file size used. This file size was swept from sizes $24 \mathrm{KBytes}$ to $56 \mathrm{KBytes}$ in order to verify the results over different operating conditions.

TABLE II. INTERFERENCE SYSTEM MODEL SPECIFICATIONS

\begin{tabular}{|l|c|}
\hline \multicolumn{1}{|c|}{ Parameter } & Value \\
\hline Distance between Interference Nodes & $4 \mathrm{~m}$ \\
\hline Inter-Request Time & $0.5 \mathrm{~s}$ \\
\hline Transmission Data Rate & $54 \mathrm{Mbps}$ \\
\hline Application Layer Protocol & FTP \\
\hline
\end{tabular}

\section{System Performance Metrics}

For each simulation, three network criteria were measured, and compared across all three systems, namely the single channel systems and their corresponding PRP system. The three network performance criteria were latency, jitter, and maximum packet end-to-end delay.

Latency: Latency here serves to measure the overall performance of the system with and without PRP. It is the average end-to-end delay of all the packets sent over all the sensor-actuator pairs.

Jitter: It is defined as the standard deviation of the end-toend delay of all the sent packets. 33 seeds were simulated, but the standard deviation was calculated for every sensoractuator pair instead of the end-to-end delay. This metric would show how much the delay fluctuates around the mean.

Maximum Packet End-to-End Delay: This metric is defined as the maximum end-to-end delay of all the packets sent for a given seed.

Maximum packet end-to-end delay serves to determine whether or not the system had any packet losses. If a packet were delayed for a period greater than the sampling period (40ms), it would be considered lost. Consequently, the packet with maximum end-to-end delay was observed and compared to $90 \%$ of the sampling period $(36 \mathrm{~ms})$. This would leave space for some margin of error. The packet would be considered lost if its total end-to-end delay exceeds $90 \%$ of the sampling period. Accordingly, the interference file size was swept over the range that showed end-to-end delays close to this failure threshold. This would show how PRP improves the system around this critical region.
For a 95\% confidence analysis, 33 seeds were run for every interference file. For every separate sensor-actuator pair, the system performance metrics were calculated for channel 1 alone, channel 6 alone, and the PRP system. This was done for each single seed separately. A confidence analysis for all 33 seeds was then carried out. Note that all the values presented in the figures represent the upper bound of the calculated confidence interval.

\section{RESUlts AND ANALYSIS}

This section presents the results for the model with interference on channel 1 only, on channel 6 only, and on both channels. Latency, jitter, and maximum packet end-to-end delay curves are presented for all scenarios in order to analyze the effect of PRP in all cases. Note that all the latencies presented in this study include packet transmission, propagation, encapsulation, de-capsulation, as well as queuing delays.

\section{A. Interference-Free Scenario}

In order to test the general operation of the model and to establish a benchmark against which the rest of the results were compared, the proposed model (Fig. 1) was simulated but with no external interference represented by the laptops. It was shown that, as expected, the two channels exhibited similar behavior since the transmission data rates and control payloads are identical. This model was then used to test how PRP would improve the system, where the PRP delay is always the minimum delay across the two channels.

The trends of the obtained results, in this study, align with those in [19]. In both studies, the PRP system is always superior.

\section{B. Interference on Channel 1}

The scenario with interference on channel 1 only was simulated with different interference file sizes that were swept from $24 \mathrm{KBy}$ tes to $56 \mathrm{KBytes}$. The obtained results were in alignment with expectations, as the interference file size increased, the amount of interference on the corresponding control channel also increased leading to medium congestion as nodes would have to retry several times to capture the channel. This meant that the maximum packet end-to-end delay increased as the interference file size increased.

Fig. 2 shows the latency for channel 1, channel 6, and their corresponding PRP results. For the scenario where external interference was applied on channel 1 only, the PRP system clearly demonstrates better latency when compared to the single-channel system. Additionally, the latency's percentage improvement of the PRP over the channel under interference was at least $121 \%$.

Fig. 3 shows the jitter for the three systems. Similarly, the PRP system always displayed less jitter when compared to the single-channel systems. This is intuitive since PRP selects the minimum end-to-end delay on a packet-by-packet basis, therefore the deviation from the mean will always be less. 


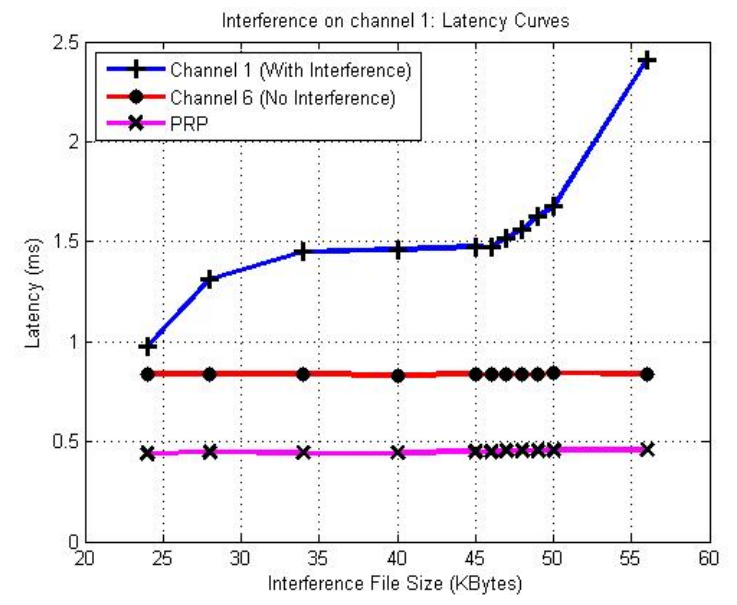

Fig. 2. Latency Curve (Interference on Channel 1)

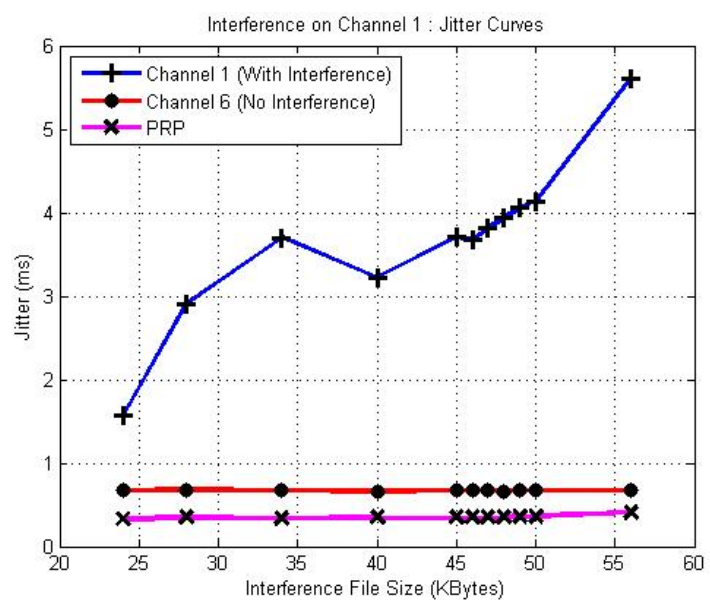

Fig. 3. Jitter Curve (Interference on Channel 1)

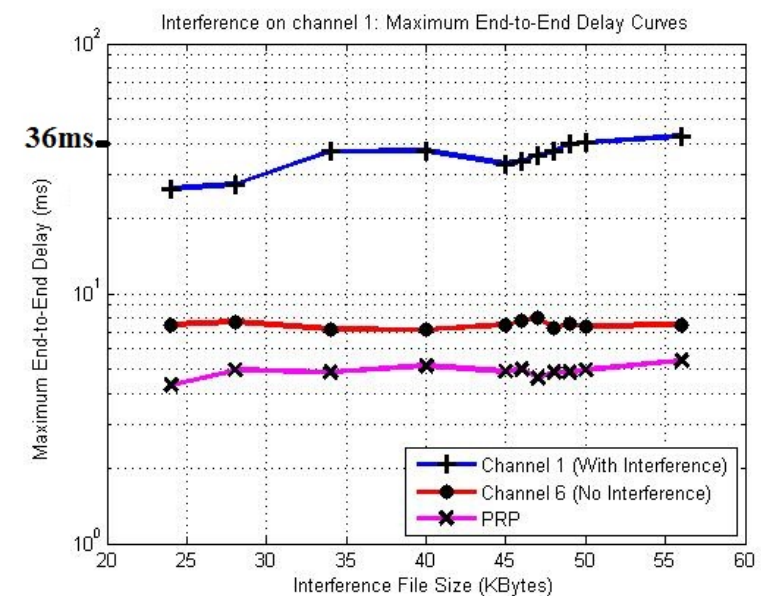

Fig. 4. Maximum End-to-End Delay Curve (Interference on Channel 1)

Fig. 4 shows the curve for the maximum packet end-to-end delay in every simulation. The threshold interference file size that caused the maximum packet end-to-end delay on channel 1 to exceed the $36 \mathrm{~ms}$ was found to be $34 \mathrm{KBytes}$, while the PRP system could theoretically operate with an infinitely large interference file size.

\section{Interference on Channel 6}

In order to validate and confirm the results that were obtained from the simulations with interference on channel 1 only, a scenario with interference being applied on channel 6 only was simulated. The results were expected to be almost identical due to the symmetry of the system. All the other parameters were set at the same values as those of the model where the interference was applied on channel 1 only. Channel allocation is therefore, the only variable from the previous model. Hence, if there were any changes caused from using the different channel, it would be observed. As expected, the results obtained from this model aligned almost exactly with those obtained from the model with interference on channel 1 only. An interference file size of $35 \mathrm{KBy}$ tes caused the maximum packet end-to-end delay on channel 6 to exceed the delay threshold of $36 \mathrm{~ms}$.

\section{Interference on both Channel 1 and Channel 6}

This scenario utilizes the same workcell as in the previous two sections; however one extra laptop pair is added to create interference on both channels 1 and 6 . Both pairs are exchanging files using FTP. The FTP file sizes used are the same as the ones used with interference applied on one channel only. The first laptop pair is transferring files on channel 1, hence causing congestion and simulating interference for this channel. Similarly, the second laptop pair is transferring FTP files on channel 6 to serve the same purpose.

Overall, this model is more detrimental to the control network than the previous two scenarios since both channels are being affected by interference. Since the interference file sizes used for both pairs are the same, it is expected to obtain almost identical end-to-end delays for the two channels. It is also expected, however, for the PRP system to have results better than any of the channels alone, since it operates on a packet-by-packet basis. Bearing in mind that since both channels have external interference, the percentage improvement provided by PRP is expected to be less than when only one channel had external interference.

Fig. 5 shows latency curves for channel 1, channel 6, and their corresponding PRP results. As expected, PRP still maintains its advantage over the other two channels.

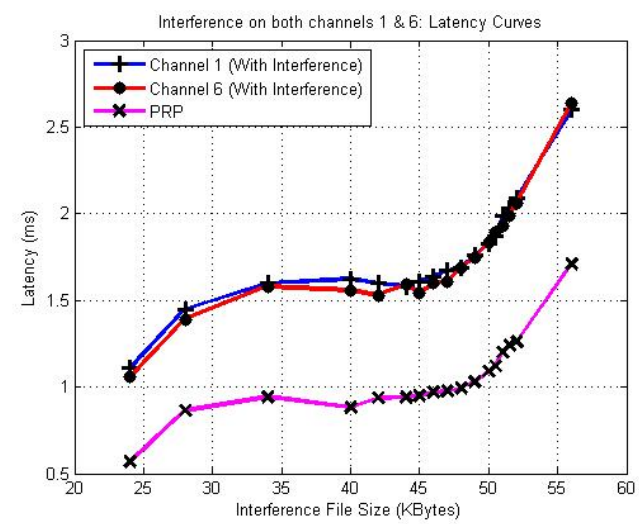

Fig. 5. Latency Curve (Interference on Channels $1 \& 6$ ) 
One difference to note here is that the delays for PRP system are increasing and no longer constant as shown in the previous model (Fig. 2) where only one of the channels was under interference. This is predictable since both channels have increasing delays due to the increasing interference file sizes. Therefore, the PRP system delay would increase accordingly.

Fig. 6 shows the jitter curves for the individual channels and their PRP results. As predicted, channels 1 and 6 show more or less similar results, and PRP clearly proves superior as well when it is compared to any channel alone.

In this study, similar to [19], the two channels show very similar trends since the interference patterns are identical in both cases. The PRP system is superior as the interference file size changes, however, unlike the previous two sections, the PRP delays increase as we increase the interference. This phenomenon was also observed in [19] and it is expected due to the nature of the PRP system. Similar to the latency curves, the PRP system does not have a constant jitter value since both channels 1 and 6 are congested. Nevertheless, the PRP system still shows a significant improvement.

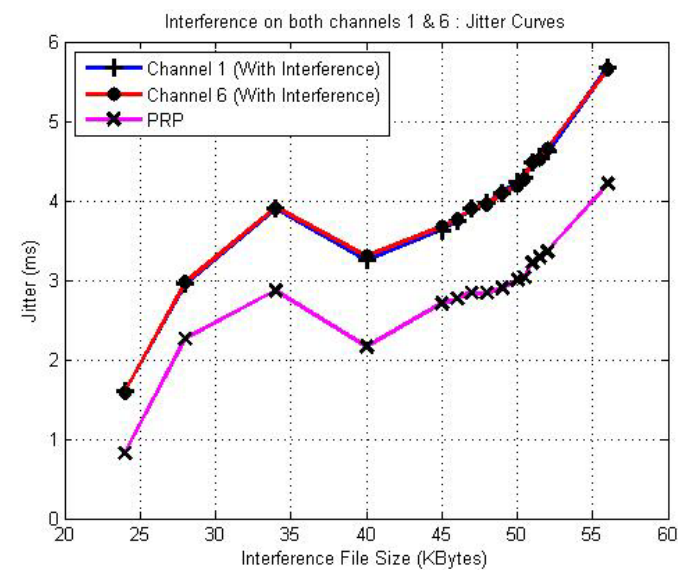

Fig. 6. Jitter Curve (Interference on Channels 1 \& 6)

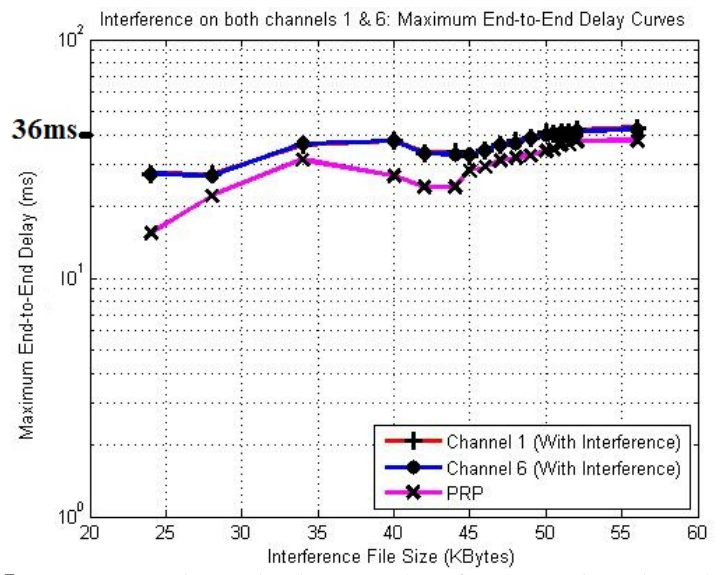

Fig. 7. Maximum End-to-End Delay Curve (Interference on Channels 1\&6)

Fig. 7 shows the maximum packet end-to-end delay of the simulation. These results show that PRP caused noticeable improvement for the maximum tolerable interference file size of the system. Any channel, when used alone, can only tolerate up to $34 \mathrm{KBy}$ tes interference file size. When PRP is introduced, this value goes up to $51 \mathrm{KBytes}$. This means that the PRP system can operate in environments with much higher external interference.

Similar to the latency curves, the PRP system does not have a constant jitter value since both channels 1 and 6 are congested. Nevertheless, the PRP system still shows a significant improvement.

Tables III and IV show the maximum end-to-end delay results for the cases where interference was applied on channel 1 only and where interference was applied on both channels $(1 \& 6)$, respectively. The three chosen values for the file sizes correspond to those at the worst percentage improvement, at the threshold, and at the best percentage improvement. For interference on one channel, from Table III, a threshold file size of $34 \mathrm{KBytes}$ caused the maximum packet end-to-end delay to exceed the delay constraint of $36 \mathrm{~ms}$. For interference on both channels, the threshold file size is 51 KBytes, as presented in Table IV. The values in Table III and Table IV are based on a $95 \%$ confidence analysis.

TABLE III. RESULTS FOR INTERFERENCE ON CHANNEL 1 ONLY

\begin{tabular}{|c|c|c|c|}
\hline $\begin{array}{c}\text { File size } \\
\text { (KBytes) }\end{array}$ & Channel 1 (ms) & Channel 6 (ms) & PRP delay (ms) \\
\hline $\mathbf{2 8}$ & {$[25.71 ; 27.31]$} & {$[6.11 ; 7.7]$} & {$[3.66 ; 4.96]$} \\
\hline $\mathbf{3 4}$ & {$[35.46 ; 37.12]$} & {$[5.69 ; 7.21]$} & {$[3.57 ; 4.86]$} \\
\hline $\mathbf{4 9}$ & {$[37.72 ; 39.54]$} & {$[5.86 ; 7.63]$} & {$[3.78 ; 4.85]$} \\
\hline
\end{tabular}

TABLE IV. RESULTS FOR INTERFERENCE ON BOTH CHANNELS ( 1 \& 6 )

\begin{tabular}{|c|c|c|c|}
\hline $\begin{array}{c}\text { File size } \\
\text { (KBytes) }\end{array}$ & Channel 1 (ms) & Channel 6 (ms) & PRP delay (ms) \\
\hline $\mathbf{2 4}$ & {$[24.89 ; 27.32]$} & {$[24.88 ; 27.39]$} & {$[13.84 ; 15.4]$} \\
\hline $\mathbf{5 1}$ & {$[39.86 ; 40.94]$} & {$[39.35 ; 40.6]$} & {$[34.75 ; 36.28]$} \\
\hline $\mathbf{5 2}$ & {$[40.61 ; 41.81]$} & {$[39.93 ; 41.29]$} & {$[35.97 ; 37.6]$} \\
\hline
\end{tabular}

Table $\mathrm{V}$ shows the worst and best percentage improvements for all the metrics in addition to the percentage improvement at the threshold for the maximum end-to-end delay. All percentages represent the improvement of PRP over the channel under interference. Note that, for the scenarios where interference was applied on only one channel, the PRP system's Maximum End-to-End delay will never experience any increase hence, the N/A percentage improvement in Table V.

TABLE V. System Percentage ImPROVEMENT (\%)

\begin{tabular}{|l|c|c|c|c|c|c|}
\hline & \multicolumn{3}{|c|}{$\begin{array}{c}\text { Interference on one } \\
\text { channel }\end{array}$} & \multicolumn{3}{c|}{$\begin{array}{c}\text { Interference on two } \\
\text { channels }\end{array}$} \\
\cline { 2 - 7 } & Worst & Threshold & Best & Worst & Threshold & Best \\
\hline Latency & 121 & N/A & 425 & 54.39 & N/A & 85.43 \\
\hline Jitter & 376 & N/A & 1268 & 30.53 & N/A & 95.12 \\
\hline Maximum & 450.4 & 663.37 & 714.43 & 11.17 & 12.98 & 77.27 \\
\hline
\end{tabular}

\section{CONCLUSION}

The concept of PRP was previously introduced in several studies. PRP is an attractive subject for study in industrial applications due to its high performance advantages and interference immunity. 
The PRP-WLAN system investigated in this study used an interference scheme that employed laptops exchanging files using FTP on the same channels utilized by the sensoractuator pairs in a workcell. The interference file sizes were swept simulating a change in the intensity of the interference. In this context, the performance of the PRP system was compared to that of the single-channel system. All results were based on a 95\% confidence analysis. This study shows that PRP is superior to a single-channel system with regards to latency, jitter, and the maximum packet end-to-end delay. The PRP system showed no dropped or over-delayed packets at all when only one channel was subjected to interference. If interference is introduced on both channels, the PRP system starts to experience over-delayed packets when the interference file size is greater than $51 \mathrm{KBy}$ tes compared to $34 \mathrm{KBytes}$ for the single-channel system.

For the scenarios where interference was applied on one channel only and where interference was applied on both channels, PRP proves to be better with regards to latency, jitter, and maximum packet end-to-end delay. As for the scenario where only one channel (either 1 or 6 ) is under interference, the latency improved by at least $121 \%$, the jitter improved by at least $376 \%$, while the maximum packet endto-end delay attained an improvement of at least $450.4 \%$. For the scenario where both channels (1 and 6) are under interference, the latency improved by at least $54.39 \%$, the jitter improved by at least $30.53 \%$, while the maximum packet end-to-end delay attained an improvement of at least $11.17 \%$. Note that all presented latencies in this study include: packet propagation, transmission, encapsulation, de-capsulation, and queuing delays and are based on a $95 \%$ confidence analysis.

\section{REFERENCES}

[1] F.L-Lian, J.R. Moyne, and D.M. Tilbury, "Networked Control Systems Toolkit: A Simulation Package for Analysis and Design of Control Systems with Network Communication," Tech. Rep., UM-ME-01-04, July 2001. Available: http://www.eecs.umich.edu/ impact

[2] T. Skeie, S. Johannessen, and C. Brunner, "Ethernet in Substation Automation," IEEE Control Syst. Vol. 22, no. 3, June 2002, pp. 43-51.

[3] J. Nilsson, Real-Time Control Systems with Delays, $\mathrm{PhD}$ thesis, Department of Automatic Control, Lund Institute of Technology, Lund, Sweden, 1998.

[4] J.P. Thomesse. "Fieldbus Technology in Industrial Automation", Proceedings of the IEEE, Vol. 93, No. 6, June 2005, pp. 1073-1101.

[5] F.L-Lian, J.R. Moyne, and D.M. Tilbury, "Performance Evaluation of Control Networks: Ethernet, ControlNet, and DeviceNet," IEEE Control Systems Magazine, Vol. 21, No. 1, pp. 66-83, February 2001.

[6] ODVA, "Volume 2: EtherNet/IP Adaptation on CIP," Available:http://www.odva.org/10_2/03_events/03_ethernethomepage.htm

[7] T.K. Refaat, R.M. Daoud, H.H. Amer and E.A. Makled, "WiFi implementation of Wireless Networked Control Systems", Proceedings of the 7th IEEE International Conference on Networked Sensing Systems, INSS, Kassel-Germany, June 2010.

[8] IEEE 802.11 Std.

[9] R. Steigmann and J. Endresen, "Introduction to WISA: WISA Wireless Interface for Sensors and Actuators", $A B B$, July 2006.

[10] ABB, "Technical Description WISA Wireless Interface for Sensors and Actuators: Planning Installation and Commissioning Guidelines," March 2009.

[11] IEEE 802.15.1 Std.

[12] IEEE 802.15.4 Std.

[13] E.E. Abdel Reheem, Y.I. El Faramawy, H.H. Halawa, M.A. Ibrahim, A. Elhamy, T.K. Refaat, R.M. Daoud, and H.H. Amer, "On the Effect of Interference on Wi-Fi-Based Wireless Networked Control Systems", Proceedings of the 8th IEEE Conference on Communication Systems, Networks and Digital Signal Processing CSNDSP, Poznan-Poland, July 2012.

[14] D.G. Brennan, "Linear diversity combining techniques," Proceedings of the IRE, vol.47, no.1, pp.1075-1102, June 1959.

[15] H. Beikirch, M. Voss and A. Fink; "Redundancy Approach to Increase the Availability and Reliability of Radio Communication in Industrial Automation"; Proceedings of the 14th IEEE International Conference on Emerging Technologies and Factory Automation ETFA, MallorcaSpain, September 2009.

[16] H. Kirrmann, M. Hansson and P. Muri; "IEC 62439 PRP: Bumpless recovery for highly available, hard real-time industrial networks"; Proceedings of the 12th IEEE International Conference on Emerging Technologies and Factory Automation ETFA, Patras-Greece, September 2007.

[17] M. Rentschler and P. Laukemann; "Towards a Reliable Parallel Redundant WLAN Black Channel"; Proceedings of the 9th IEEE International Workshop on Factory Communication Systems WFCS, Lemgo/Detmold-Germany, May 2012.

[18] M. Rentschler and P. Laukemann; "Performance Analysis of Parallel Redundant WLAN"; Proceedings of the 17th IEEE International Conference on Emerging Technologies and Factory Automation ETFA Krakow-Poland, September 2012.

[19] M. Rentschler, O.A. Mady, M.T. Kassis, H.H. Halawa, T.K. Refaat, R.M. Daoud, H.H. Amer, and H.M. ElSayed, "Simulation of Paralle Redundant WLAN with OPNET"; Proceedings of the $18^{\text {th }}$ IEEE International Conference on Emerging Technologies and Factory Automation ETFA, Cagliari-Italy, September 2013.

[20] C.M.D. Viegas, S. Sampaio, F. Vasques, P. Portugal and P. Souto, "Assessment of the Interference caused by uncontrolled traffic sources upon real-time communication in IEEE 802.11-based mesh networks," Proceedings of the 9th IEEE International Workshop on Factory Communication Systems (WFCS), Lemgo/Detmold-Germany, May 2012.

[21] G. Boggia, P. Camarda, V. Divittorio and L.A. Grieco, "A simulation-based performance evaluation of Wireless Networked Control Systems", Proceedings of the IEEE International Conference on Emerging Technologies and Factory Automation ETFA, MallorcaSpain, September 2009.

[22] T.K. Refaat, R.M. Daoud and H.H. Amer, "Fault-Tolerant Controllers in Wireless Networked Control System using 802.11g”, Proceedings of the IEEE International Conference on Industrial Technology ICIT, Athens-Greece, March 2012. 